\section{Réguler l'expression d'un transgène thérapeutique par un régime alimentaire carencé en un acide aminé indispensable}

Alain Bruhat ${ }^{1}$, Cédric Chaveroux ${ }^{1}$, Valérie Carraro ${ }^{1}$, Céline Jousse $^{1}$, Julien Averous ${ }^{1}$, Anne-Catherine Maurin ${ }^{1}$, Laurent Parry ${ }^{1}$, Florent Mesclon ${ }^{1}$, Yuki Muranishi ${ }^{1}$, Patrick Baril ${ }^{2}$, Anh Do Thi ${ }^{3}$, Philippe Ravassard ${ }^{3}$, Jacques Mallet ${ }^{3,4}$, Pierre Fafournoux ${ }^{1}$

> La thérapie génique consiste à apporter un gène au sein des cellules pour soigner ou prévenir des maladies. Ce gène peut alors remplacer un gène défectueux, dans le cas d'une maladie génétique, ou permettre de fabriquer et diffuser localement une molécule thérapeutique. Une des limites importantes de l'utilisation de la thérapie génique est l'absence de contrôle de l'expression du transgène (le gène introduit dans l'organisme) [1]. Les seuls systèmes inductibles actuels décrits chez la souris nécessitent l'expression de protéines régulatrices exogènes (récepteurs) ainsi que des molécules chimiques inductrices (comme la doxycycline ${ }^{1}$, l'ecdysone ${ }^{2}$, la rapamycine ${ }^{3}$, etc.). Or, les effets secondaires potentiels de ces systèmes empêchent leur utilisation en médecine humaine.

Nous présentons ici un système, que nous venons de mettre au point, dans lequel l'expression d'un transgène est contrôlée par la consommation d'un régime alimentaire particulier où un acide aminé indispensable (AAI) est absent [2].

\footnotetext{
${ }^{1}$ Molécule de la famille des cyclines utilisée comme médicament antibiotique.

${ }^{2}$ Hormone stéroïde d'insecte.

${ }^{3}$ Molécule produite par la bactérie Streptomyces hygroscopicus possédant des propriétés immunosuppressives.
}

\section{De la nutrition à la thérapie génique}

\section{L'homéostasie des acides aminés}

Chez les adultes sains en bonne santé, neuf acides aminés (valine, isoleucine, leucine, lysine, méthionine, phénylalanine, thréonine, tryptophane, histidine) sont indispensables (AAI), c'est-à-dire qu'ils ne peuvent pas être synthétisés de novo par l'organisme et doivent être impérativement apportés par l'alimentation. De plus, les acides aminés ne possèdent pas de système de stockage dédié, comme c'est le cas pour les lipides (triglycérides du tissu adipeux) ou le glucose (glycogène). Par conséquent, en cas de déficit alimentaire en AAI, des mécanismes permettant d'adapter l'homéostasie des acides aminés à cette nouvelle situation nutritionnelle sont obligatoires [3]. Une situation nutritionnelle très fréquente chez les omnivores à l'état sauvage est la consommation d'un régime alimentaire déficient en un ou plusieurs AAI. Un animal n'ayant à sa disposition qu'une seule source protéique d'origine végétale a de fortes chances d'être carencé en un ou plusieurs AAI [3]. II a été montré, chez les rongeurs et les omnivores (rat, souris, cochon, poulet, etc.), que la consommation d'un tel régime entraîne une diminution rapide et nette de la prise alimentaire. Ce phénotype résulte de la détection
${ }^{1}$ Unité de nutrition humaine, UMR 1019, INRA de Clermont-Theix, Université ClermontFerrand 1, 63122 Saint Genès Champanelle, France ;

${ }^{2}$ Centre de biophysique moléculaire, CNRS UPR4301, Université d'Orléans, Inserm, Orléans, France ;

${ }^{3}$ CNRS UMR7225, Inserm U1127, Université

Pierre et Marie Curie, Institut du Cerveau et de la Moelle (ICM), Biotechnology and Biotherapy Team, Paris, France ;

${ }^{4}$ Department of neurological surgery, University of California San Francisco (UCSF), San Francisco, CA, États-Unis. pierrre.fafournoux@inra.fr

par le cerveau de la diminution de la concentration plasmatique de l'acide aminé limitant. Ce comportement permet de rejeter un régime déséquilibré et de favoriser la recherche d'une nourriture équilibrée [4]. Cet exemple montre que l'organisme est capable de détecter le manque d'acide aminé et de réguler certaines fonctions physiologiques permettant l'adaptation à une nouvelle situation nutritionnelle. Pour cela, il active une voie de signalisation spécifique qui entraîne la régulation de l'expression de certaines protéines impliquées dans les mécanismes d'adaptation à des apports nutritionnels inadaptés.

La voie de signalisation GCN2/elF2a/ ATF4 est activée par un manque d'acide aminé

Notre équipe a démontré qu'un manque d'AAl peut réguler des fonctions physiologiques au travers de la régulation de l'expression de gènes cibles. Nous avons identifié un élément de réponse aux acides aminés (AARE) localisé dans les promoteurs de ces gènes [5]. Nous avons également contribué à la caractérisation de la voie de signalisation activée par la carence en AAl et conduisant à la surexpression de gènes cibles. Le détecteur de la carence en 
A

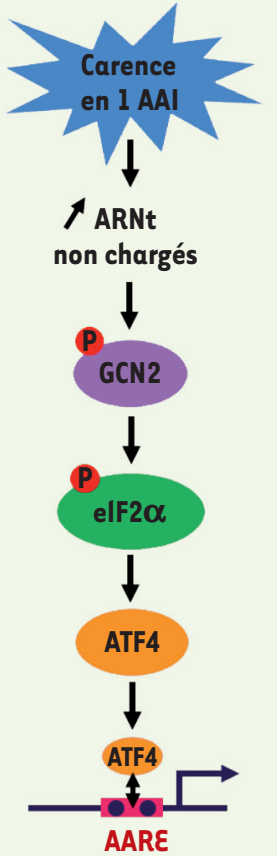

Gènes cibles spécifiques (TRB3, CHOP, ATF3, etc.)
B

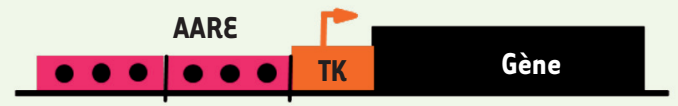

à un déficit en acides aminés. Cette protéine kinase possède un domaine homologue à l'histidyl-ARNt synthétase lui permettant de lier les ARN de transfert non-chargés, dont la concentration augmente à I'intérieur de la cellule lors d'une carence en un acide aminé. II résulte de cette liaison un changement conformationnel permissif conduisant à l'auto-activation de GCN2 qui va alors phosphoryler la sousunité $\alpha$ du facteur d'initiation de la traduction elF2. La phosphorylation d'elF2 $\alpha$ va avoir pour conséquence une inhibition de la formation du complexe d'initiation de la traduction, entraînant une forte inhibition de la synthèse protéique. Dans ces conditions, et grâce à une structure particulière de sa région 5' non traduite, la traduction du messager du facteur de transcription ATF4 est augmentée. ATF4 peut alors induire la transcription de gènes cibles possédant dans leur promoteur des éléments de réponse aux acides aminés (amino acid response element ou AARE). AAI : acide aminé indispensable; TRB3 : Tribbles homolog 3 ; CHOP: C/EBP (CCAAT-enhancer-binding proteins) homologous protein; ATF3: activating transcription factor 3. B. La construction utilisée contient un promoteur constitué de plusieurs copies de la séquence AARE précédant et d'un site d'initiation de la transcription TK (thymidine kinase). II dirige l'expression d'un transgène. acide aminé est la protéine kinase GCN2 (general control nonderepressible 2) qui, en phosphorylant le facteur d'initiation de la traduction elF $2 \alpha$ (eukaryotic initiation factor $2 \alpha$ ), va induire la traduction du facteur de transcription ATF4 (activating transcription factor $4 ; \quad \rightarrow$ Voir la Nouvelle de voir Figure 1 pour A. Bruhat et al., $\mathrm{m} / \mathrm{s}$ plus de détails) $\mathbf{n}^{\circ} \mathbf{1 2}$, décembre 2015 , $[6](\rightarrow)$. page 1057

Nous avons également montré que cette voie est fonctionnelle in vivo. Elle est activée suite à la consommation d'un régime déséquilibré en $A A I$ et permet l'adaptation de certaines fonctions physiologiques. Par exemple, l'activation de GCN2 dans le cerveau, met en route un mécanisme d'inhibition de la prise alimentaire chez la souris [7].

\section{Concept}

Nous avons utilisé les propriétés de cette voie de signalisation pour générer un système de régulation constitué d'un promoteur artificiel, fortement inductible par une limitation en AAI, et d'un inducteur, un repas carencé en un $\mathrm{AAI}$ [2]. Ce système est fonctionnel in vivo, et pourra être utilisé pour contrôler l'expression d'un transgène dans des protocoles de recherche ou dans des essais de thérapie génique.

\section{Promoteur}

Le promoteur utilisé comprend des éléments de réponse aux acides aminés (AARE) du gène TRB3 (Tribbles homolog 3) fusionnés au promoteur minimum thymidine kinase (TK), qui apporte un site d'initiation de la transcription. Plusieurs copies de l'AARE ont été clonées en tandem afin d'obtenir une induction maximale. $\varepsilon n$ réponse à une carence en un AAI, la voie de signalisation GCN2/ATF4 est induite, ATF4 est alors surexprimé et va se fixer sur les copies de l'AARE pour activer la transcription.

\section{Inducteur}

L'activation transcriptionnelle du promoteur décrit ci-dessus est réali- sée en provoquant une diminution de la concentration sanguine en un AAl suite à l'ingestion d'un repas carencé. Il faut préciser que la concentration sanguine en acides aminés est particulièrement sensible à un régime déséquilibré en AAI, alors qu'elle n'est que peu affectée par un jeûne total (pour explications voir [3]).

Un tel régime doit être administré après un jeûne de courte durée et peut être apporté sous forme d'un «cocktail » d'acides aminés libres ou d'un repas complet dans lequel les protéines sont remplacées par un mélange d'acides aminés libres. La déplétion sanguine en AAl survient quelques minutes après la prise du repas carencé, et peut être arrêtée très rapidement par l'administration de I'AAl manquant. La carence en n'importe quel AAl est fonctionnelle. Il est donc possible, pour un traitement à moyen ou long terme, d'alterner la carence en différents AAl de façon à éviter des manques nutritionnels. 


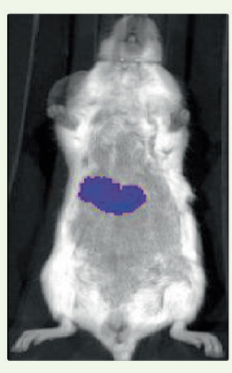

Ctrl

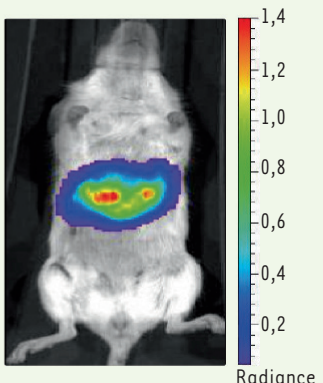

- -lle

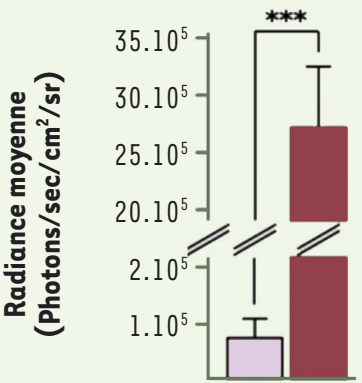

Ctrl-Ile
2XAARE-TK-LUC croissance tumorale [2]. II est important que son expression soit localisée sur le site de la tumeur et très finement régulée. En effet, (1) TRAIL pourrait être potentiellement toxique pour des cellules non-quiescentes (cellules souches neurales) en cas d'administration forte et prolongée ; (2) de plus, la protéine TRAIL a une demi-vie très courte.

\section{Caractéristiques et avantages}

Notre système d'expression génique régulé par la nutrition possède plusieurs avantages: (1) il utilise des mécanismes moléculaires endogènes. Par conséquent, son induction n'est pas conditionnée par l'expression de protéines régulatrices exogènes susceptibles de générer des problèmes immunologiques chez l'hôte; (2) il ne présente aucune toxicité. Nous avons vérifié que l'alternance entre des régimes carencés en différents $A A I$ n'affecte pas la masse maigre; (3) il présente une réversibilité totale et rapide de l'expression du transgène permettant une délivrance de la protéine thérapeutique par «pulse »; (4) l'expression du transgène peut être répétée dans le temps via la consommation alternée de régimes carencés en différents AAI ; (5) la chute de concentration plasmatique de l'acide aminé limitant se répercutera au-delà de la barrière hémato-encéphalique et pourra cibler directement un site d'intérêt dans le cerveau.

\section{En conclusion}

La découverte d'un moyen simple, fiable et sans risque pour réguler l'expression des gènes constitue une avancée essentielle dans le domaine de la thérapie génique et ses multiples champs d'application. En effet, l'expression du gène-médicament nécessite souvent une régulation fine et un dosage précis. Ce système va ainsi permettre de mettre en place de nouveaux traitements par thérapie génique jusque-là inenvisageables [8]. $\diamond$

Tuning transgene expression with an artificial diet: a compelling resource in gene therapy 
A

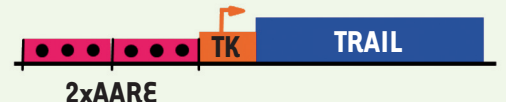

B

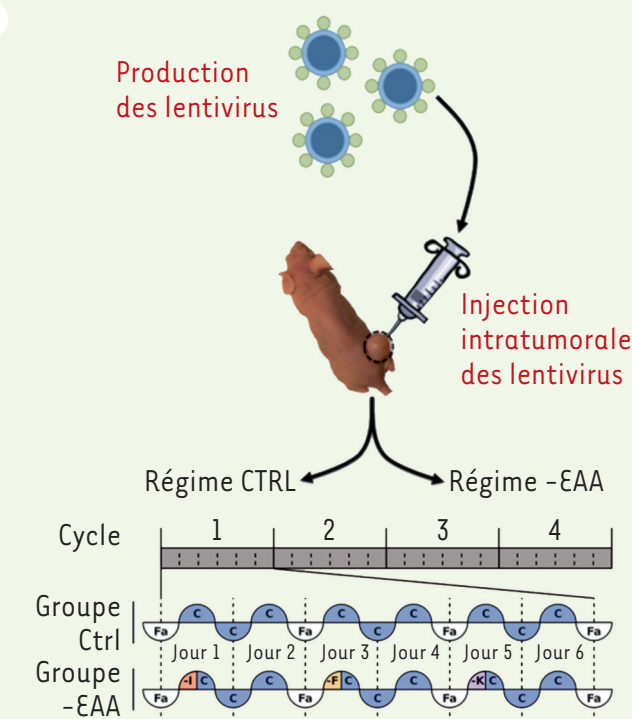

C

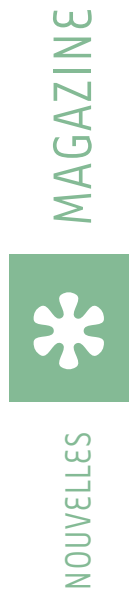

Figure 3. Inhibition de la croissance tumorale par l'expression régulée de TRAIL (tumor-necrosis-factor related apoptosis inducing ligand). A. Schéma de la construction utilisée. AARE : amino acid response element ; TK : thymidine kinase. B. Protocole expérimental. Les cellules Gli36-Luc ont été implantées par voie sous-cutanée chez la souris nude. Une semaine après, les injections intra-tumorales du lentivirus LV-AARE-TRAIL ont été réalisées. Les souris ont ensuite été soumises au protocole nutritionnel. Elles ont été soumises à la séquence suivante : $12 \mathrm{~h}$ de jeûne (couleur blanche, $\mathrm{Fa}$ ), $6 \mathrm{~h}$ d'alimentation avec un régime carencé (couleur rouge, $-\mathrm{I}$; couleur jaune, $-\mathrm{F}$; couleur violette, $-\mathrm{K}$ ) et $30 \mathrm{~h}$ de période de récupération avec un régime contrôle (couleur bleue, $\mathrm{C}$ ). Cette séquence nutritionnelle est répétée pendant deux semaines. Pour le groupe contrôle (Ctrl), les régimes inducteurs carencés $(-I,-F,-K)$ ont été remplacés par un régime contrôle. $E A A$ : essential amino acid; $I$ : isoleucine ; $F$ : phénylalanine; $K$ : lysine. C. Suivi de la croissance tumorale. La croissance tumorale a été suivie par imagerie bioluminescente le premier jour après implantation des cellules (TO) puis chaque semaine. Une photo représentative des tumeurs 3 semaines post-implantation est montrée.

\section{REMERCIEMENTS}

Ce travail a été financé par l'ANR «Programmes Émergence »(ANR-12-EMMA-0024) et STRESSOR (ANR-12-BSV2-0025-03).

\section{LIENS D'INTÉRÊT}

Les auteurs déclarent n'avoir aucun lien d'intérêt concernant les données publiées dans cet article.

\section{RÉFÉRENCES}

1. Misra S. Human gene therapy: a brief overview of the genetic revolution. J Assoc Phys India 2013 ; 61 : 127-33.
2. Chaveroux C, Bruhat A, Carraro V, et al. Regulating the expression of therapeutic transgenes by controlled intake of dietary essential amino acids. Nat Biotechnol 2016 ; 34 : 746-51.

3. Chaveroux C, Lambert-Langlais S, Cherasse $Y$, et al. Molecular mechanisms involved in the adaptation to amino acid limitation in mammals. Biochimie 2010 ; $92: 736-45$.

4. Maurin AC, Jousse C, Averous J, et al. The GCN2 kinase biases feeding behavior to maintain amino acid homeostasis in omnivores. Cell Metab $2005 ; 1$ : 273-7.

5. Bruhat A, Jousse C, Carraro V, et al. Amino acids control mammalian gene transcription: activating transcription factor 2 is essential for the amino acid responsiveness of the CHOP promoter. Mol Cell Biol $2000 ; 20: 7192-204$.

6. Bruhat A, Jousse C, Carraco V, et al. L'activation de la voie elF2 $\alpha$-ATF4, une réponse adaptative au stress cellulaire. Med Sci (Paris) 2015 ; 31 : 1057-60.

7. Maurin AC, Benani A, Lorsignol A, et al. Hypothalamic elF2 $\alpha$ signaling regulates food intake. Cell Rep 2014 ; $6: 438-44$.

8. Anguela XM, High KA. An edible switch for gene therapy. Nat Biotechnol $2016 ; 34: 824-5$.

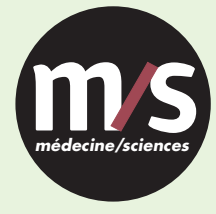

Tarifs d'abonnement $\mathrm{m} / \mathrm{s}-2017$

Abonnez-vous

à médecine/sciences
$>$ Grâce à $m / s$, vivez en direct les progrès des sciences biologiques et médicales

Bulletin d'abonnement page 202 dans ce numéro de $\mathrm{m} / \mathrm{s}$

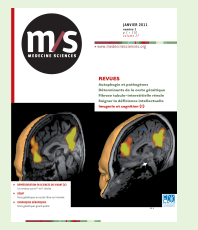

